\title{
Le face à face verbal et la puissance de la persuasion dans la pièce "Quai ouest" de Bernard-Marie Koltès
}

\author{
Alaaedin Baheidin Alaaedin* \\ alaabahi364@gmail.com
}

\section{Résumé}

Bernard-Marie Koltès est né en 1948 à Metz et mort en 1989 du SIDA à l'âge de quarante et un ans. Il a écrit six pièces très importantes. Sa carrière qui a duré une dizaine d'années a suffi pour que son nom soit connu dans le monde entier et surtout au milieu du monde théâtral que: "ses textes recèlent quelque chose de frappant" (Vigeant, 1990: 35). Son théâtre représente une tentative continuelle et vaine pour créer une communication entre les hommes.

Donc, notre étude sera concentrée sur les dialogues chargés de confrontations verbales et langagières à la lumière de la rhétorique persuasive. Nous allons traiter également le sujet du silence d'Abad qui n'est pas en effet ni sourd ni muet, mais ne parle jamais sur la scène. Mais le plus étonnant dans cette pièce que la plupart des personnages ne parlent qu'avec Abad, car il accepte leurs paroles et ne les refuse pas. Ce mutisme a un rôle

* Professeur adjoint à la faculté des Lettres, Université du Sud de la vallée.

(Le face à face verbal et la puissance ...) Dr. Alaaedin Baheidin Alaaedin 
symbolique dans la pièce, qui sera un thème majeur dans notre étude.

\section{Mots-clés: le face à face, la persuasion, la communication}

\section{Introduction}

Bernard-Marie Koltès est né en 1948 à Metz et mort en 1989 du SIDA à l'âge de quarante et un ans. Il a écrit six pièces très importantes. Sa carrière qui a duré une dizaine d'années a suffi pour que son nom soit connu dans le monde entier et surtout au milieu du monde théâtral que: "ses textes recèlent quelque chose de frappant" (Vigeant, 1990: 35). Son théâtre représente une tentative continuelle et vaine pour créer une communication entre les hommes.

Dès le début de la vingtaine, il commence à écrire des textes dramatiques sur commande. Son essor réel commence avec l'apparition de "La Nuit juste avant les forêts" après une période de souffrance et de l'expérience de la drogue et à la suite d'une tentative de suicide. Ensuite, les œuvres se succèdent avec un grand succès. Avec "Combat de nègre et de chiens", Koltès a réalisé un grand éclat. En 1982, il commence à écrire "Quai ouest" qui sera la base de notre étude, la pièce a été créée en 1986 par Patrice Chéreau, mais sans grand succès. En 1988, Koltès se tourne vers le comique qui a caractérisé son "Retour au désert". La dernière grande pièce de Koltès était "Roberto Zucco", cette 
pièce qui tourne autour de l'évasion d'un aliéné mental italien de vingt-six ans, ne sera créée qu'un an après sa mort.

Les pièces de Koltès sont liées étroitement au mouvement du théâtre français des années 1980. Son théâtre se présente sous formes de textes autonomes. Parmi les thèmes essentiels de son théâtre: le manque de communication, la confrontation avec l'autre, l'inconnu, les relations sentimentales, l'échange et le commerce verbal...etc.

La pièce "Quai ouest" est une pièce violente, dans laquelle, nous remarquons trois morts et un viol d'une jeune fille, mais la violence ne se trouve qu'au dernier moment de la pièce. La violence corporelle reste cachée derrière la confrontation verbale des personnages, cette confrontation conversationnelle se transforme à la fin en violence corporelle.

La violence dans "Quai ouest" avec ses deux formes aboutit à des conséquences dramatiques provenant de cette confrontation entre deux monde tout à fait opposés, et cela se manifeste dès le début de la pièce, qui s'ouvre sur une rencontre entre deux personnages, chacun appartient à un monde complètement différent.

La pièce "Quai ouest" a une dimension persuasive très claire. L'analyse de la pièce consiste sur la difficulté de la communication dû au sophisme et les arguments fallacieux, où les désirs s'affrontent, symbolisés par des objets qui se trouvent dans 
les mains des personnages comme: un briquet, des clés de voitures, une montre Rolex...

Donc, notre étude sera concentrée sur les dialogues chargés de confrontations verbales et langagières à la lumière de la rhétorique persuasive. Nous allons traiter également le sujet du silence d'Abad qui n'est pas en effet ni sourd ni muet, mais ne parle jamais sur la scène. Mais le plus étonnant dans cette pièce que la plupart des personnages ne parlent qu'avec Abad, car il accepte leurs paroles et ne les refuse pas. Ce mutisme a un rôle symbolique dans la pièce, qui sera un thème majeur dans notre étude.

\section{I- Le théâtre koltésien}

Dans un entretien avec Hervé Guibert, dans Le Monde, Bernard-Marie Koltès a expliqué sa carrière d'écriture théâtrale en montrant les notions et les caractéristiques de sa langue et de sa dramaturgie. A première vue, noue remarquons que Koltès accorde une grande importance aux monologues et surtout dans ses premières pièces en disant que:

"Mes premières pièces n'avaient aucun dialogue, exclusivement des monologues. Ensuite, j'ai écrit des monologues qui se coupaient. Un dialogue ne vient jamais naturellement. Je verrais volontiers deux personnes face à face, l'une exposer son 
affaire, et l'autre prendre le relais." (Guibert, Le Monde, 1983).

Nous remarquons que Koltès accorde une importance majeure pour les situations dramatiques, dans lesquelles, nous trouvons deux personnages se rencontrent face à face, Jean-Pierre Ryngaert explique que:
"Koltès affectionne les situations de vis-à- vis, les duels et les duos, rarement pour engager les personnages dans des conflits qui noueraient une intrigue ou feraient avancer l'action." (Ryngaert, 2011: 287).

La langue koltésienne ne s'intéresse pas au naturalisme, mais elle reste tout près du réel et des sensations des personnages où nous apercevons que: "Koltès investirait beaucoup de luimême dans la création de ses personnages." (Purkhardt, Jeu, 1998: 92), cela signifie que l'auteur se trouve dans ses textes d'une façon plus ou moins directe, dans ses personnage. C'est une langue qui a toutes les caractéristiques, elle recule, se répète et déclare d'une façon étonnante les limites de la pensées de ses personnages qui se rencontrent l'un en face à face avec l'autre en exprimant leurs désirs inapaisables, donc, c'est une: "œuvre plus dérangeante que désespérée, plus cynique que désolée..." (Purkhardt, Jeu, 1998: 72). 


\section{II- "Quai ouest": pièce de la confrontation}

La naissance de la pièce "Quai ouest" était déclarée au cours d'une entrevue avec Bernard-Maris Koltès, il racontait qu'il a passé une nuit, caché, dans le hangar déserté d'un ancien port de New York, cette histoire est citée dans un entretien accordé par Patrice Chéreau à Didier Méreuze:

"Dès que l'on y pénètre, on se rend compte que l'on se trouve dans un coin privilégié $d u$ monde... un lieu ou l'ordre normal n'existe pas, mais où un autre ordre, très curieux, s'est créé." (Mereuze, 1990: 117).

Ce lieu décrit par Koltès avait toutes les caractéristiques d'un excellent lieu dramatique, notamment, si cette rencontre entre ces personnages n'était pas déjà prédisposée, ceux-ci sont mis en situation soudainement. C'est la source et l'histoire de la création de la pièce "Quai ouest" dans laquelle l'intrigue dramatique s'est développée dans un vieux quartier d'une cité occidentale, à l'intérieur d'un hangar assiégé entre une route et un fleuve où tous les moyens de transport sont coupés avec la ville, ainsi que les services municipaux:

"Quai ouest représente un endroit plongé dans la pénombre, où l'être et le paraître s'affrontent, où la vie et la mort se conjuguent à la lumière et à l'obscurité, et où le voir et le non-voir sont des indicateurs 
d'un profond bouleversement." (Hufmann, 1999: 117).

Dans "Quai ouest", Koltès a créé un espace qui s'accorde avec ses personnages, il a réussi à personnifier ce lieu selon les indications scéniques qui indiquent le changement de lumière sur cet espace scénique. C'est un jeu de lumière et d'ombre qui invite les lecteurs à réfléchir que le hangar représente une personne qui exerce une force invisible et mystique sur les autres personnages dans la pièce:

"Dans Quai ouest...c'est le fameux hangar, dont les jeux de lumière font l'objet d'une description si érotisée qu'ils en deviennent comme la métaphore muette de tous les "trafics" dont Koltès y a été le témoin sans vouloir ne rien en dire." (Hirschmuler, 2004: 17).

Tandis que Patrice Chéreau s'accorde avec l'idée que: "ce hangar, c'est presque le personnage principal de la pièce." (Chéreau, 1996: 46). Donc, le hangar à l'instar d'Abad parle sans voix, c'est: "une version moderne de l'enfer antique." (Bernard, 2010: 132). Ce hangar représente donc l'espace théâtral qui enferme les personnages qui essaient de leur tour d'en sortir, mais vainement. Nous pouvons dire que le hangar déserté et Abad représentent une image tragique de notre monde contemporain. 
Finalement, nous remarquons que la plupart des personnages dans "Quai ouest" souffrent de la pauvreté, de la haine, du manque d'espoir, du manque de confiance en euxmêmes. Mais avec ces circonstances, chaque personne a besoin de l'autre, personne n'a la puissance d'affronter son problème tout seul, c'est pourquoi, le monde de "Quai ouest" devient un monde d'échange commercial.

\section{III- Dramaturgie de la confrontation}

Il est à remarquer que Bernard-Marie Koltès aimait beaucoup les films d'action chinois "Kung-fu", où dans ces films, les scènes frappantes sont celles de duel et de la confrontation face à face, et l'attaque continue sans cesse jusqu'à la fin, cela se manifeste dans sa parole en disant:

"Les matchs de boxe, c'est un résumé de tout

l'art dramatique" (Koltès, 1999: 77).

Donc, nous pouvons dire que l'entrée de la dramaturgie koltésienne réside dans cette confrontation ou dans le face à face verbal où deux monologues se confrontent:

"Tout le théâtre de B.-M. Koltès se présente

comme un montage de situations de langage

gestualisé... de combat verbal, de combat

des idées." (Sarrazac, 2004: 108).

La dramaturgie du face à face verbal, qui provient de la confrontation des deux mondes contradictoires mène les protagonistes à une zone où la parole reste l'arme unique pour 
eux. Nous remarquons également que ce face à face précède toujours l'échange commercial, Flippo Bruschi a cité la succession logique de ces échanges qui sont:" l'échange informatif, économique et érotique" (Bruschi, 2014: 202). L'enseignement jésuite de Koltès a quitté son influence sur son écriture dramatique et sur l'importance de l'argumentation où: "L'enseignement jésuite se fonde sur la volonté de considérer un dialogue comme une vraie argumentation." (Salino, 2009: 27).

Dans le dialogue koltésien, les deux personnages qui s'affrontent sont intéressés de se persuader jusqu'à la fin de leur confrontation où: "les rapports entre les personnages sont concentrés sur le troc. La question n'est pas tellement de savoir ce qu'ils auraient à offrir, mais bien à échanger." (Vigeant, 1990: 38).

En essayant de comprendre la technique de l'écriture théâtrale de Koltès, nous remarquons que celui-ci construit ses personnages avant de créer l'intrigue de ses pièces, le monologue dans le théâtre de Koltès provient du caractère du personnage où: "quelqu'un parle et l'Autre ne lui répond pas..." (Ubersfield, 1999: 161).

L'espace scénique dans la pièce est représenté par son titre "Quai ouest", qui est un ancien hangar abandonné. Dans cet espace, nous trouvons un couple qui se rencontre par hasard, ce couple arrive de l'autre côté du hangar. C'est une rencontre entre deux mondes opposés. Le contraste entre les deux mondes 
domine la pièce, ce contraste sera concrétisé également dans l'opposition spatiale (ici et là), et l'opposition temporelle (maintenant et avant), ainsi que l'opposition des classes sociales.

Dans la pièce "Quai ouest", nous remarquons que l'auteur expose des marchandises entre les personnages, ceux-ci n'ont pas de nom comme l'espace ambigu et sombre qui les enferme, ces personnages commencent à entrer dans des duels langagiers, mais qui n'arrivent pas à la violence physique, c'est le respect qui domine leur dialogue. Avec les personnages qui ne portent pas de nom, l'espace neutre et l'absence d'action dramatique aident à créer des formes de rhétorique comme la métaphore et la comparaison. Chaque personnage déploie les efforts nécessaires pour persuader en utilisant les ficelles langagières possibles qui aident les personnages qui s'affrontent face à face.

\section{$\underline{\text { IV-Deux mondes contradictoires }}$}

Les personnages dans "Quai ouest" sont divisés en deux groupes ou deux mondes contradictoires. Avec cette confrontation sociale, commence le conflit dans la pièce et par conséquent, apparaît ce face à face verbal à cause de cette opposition qui est:

"une figure chérie des classiques. L'effet de symétrie est fondé sur l'énoncé de propositions contraires dont l'une a pour fonction de mettre l'autre en valeur." (Robrieux, 1993: 82).

(Le face à face verbal et la puissance ...) Dr. Alaaedin Baheidin Alaaedin 
La contradiction sociale de ces deux groupes se reflète également dans la contradiction de l'espace théâtral qui est considéré comme un élément nécessaire dans la dramaturgie de "Quai ouest". Marie-Paul Sébastien affirme cet avis en disant que: "La ville donne l'ordre, le monologue est sa forme dite." (Sébastien, 1999: II).

L'espace scénique dans le théâtre koltésien est complètement lié à des oppositions comme: chaud et froid, blanc et noir, mort et vivant..., ces oppositions sont liées de près ou de loin à l'espace dans ses pièces:

"Il faut des circonstances, des événements ou des lieux bien précis...; le plateau de théâtre en est un, certainement." (Koltès, 1986: 23).

Donc, le lieu et le temps représentent une image qui reflète les personnages; Marc Augé a précisé la relation forte entre l'espace et le temps en montrant que l'espace: "s'applique indifféremment à une étendue, à une distance entre deux choses ou deux points... ou à une grandeur temporelle." (Purkhardt, 1998: 91).

L'espace dans la pièce est caractérisé par l'ambiguïté. Koltès a expliqué sa notion pour le lieu dans son livre "Une part de ma vie" en disant qu': "un lieu où l'ordre normal n'existe pas, mais où un autre ordre, très curieux, s'est créé." (Koltès, 1999: 13).

(Le face à face verbal et la puissance ...) Dr. Alaaedin Baheidin Alaaedin 
La pièce commence par la présence d'un couple, la présence de deux personnages opposés a provoqué une confrontation entre deux mondes contradictoires. Affrontement entre deux personnages qui appartiennent à des milieux différents est dû à la divergence spatiale qui peut créer une rupture entre les interlocuteurs. Cette confrontation représente une séparation irréparable entre eux.

L'opposition dans la pièce se manifeste dans tous les dialogues, ainsi qu'entre les interlocuteurs et leurs relations. Dans "Quai ouest", l'espace théâtral, c'est le hangar déserté qui offre des images malheureuses de la vie des personnages:

"Si le hangar figure "un lieu de l'âme", les

personnages qui le traversent pourraient

représenter les étapes et les enjeux d'une

individuation." (Purkhardt, 1998: 91).

En ce qui concerne le lieu dans la pièce, nous avons également le fleuve qui sépare les deux mondes, le monde du hangar déserté où se déroule la pièce et la grande ville d'où arrivent Monique et Koch. Les mots dits par Monique au début de la pièce sont: "Où? Par où?, comment?, Par ici?... mais à cette heure, ici, dans ces habits!" (Koltès, Quai ouest, 1985: 11). Nous remarquons que les expressions liées à "ici" sont négatives comme: "les rats et les cafards ont pénétré ici comme des soldats vainqueurs." (Koltès, Quai ouest, 1985: 14). Inversement, les expressions liées à "là-bas" sont positives. Dans l'extrait suivant 
de Charles qui montre la vie misérable d' "ici" en montrant la contradiction entre "ici" et "là-bas":

"Charles- De l'autre côté, là-bas, c'est le haut; ici, c'est le bas; ici même, on est le bas du bas, on ne peut pas aller plus bas, et il n'y a beaucoup d'espoir de monter un peu... je préfère aller là-bas; je préfère être, làbas, le bas du haut qu'ici, le haut du bas." (Koltès, Quai ouest, 1985: 60).

Donc, nous pouvons remarquer que dans "Quai ouest": "ici" symbolise l'espace scénique, tandis que "là-bas" symbolise le "hors-scène", que nous ne voyons pas sur la scène, c'est un espace fictif.

Quant au temps, le temps joue un rôle très important dans le théâtre koltésien car: "Quand une pièce de Koltès commence, il y a aussi un "temps déjà là", celui d'une faute ou d'un manque antérieur... péché originel d'un monde livré aux lois de l'échange mercantile..." (Lazaridès, Jeu, 1999: 155).

L'opposition se trouve dans le temps comme dans le cas du lieu, ces deux éléments sont liés étroitement pour préciser les conditions et les détails des actions dramatiques car: "l'analyse de l'espace-temps met en valeur l'importance du lieu scénique, "contenant" originaire dont sont issus les personnages et qui est, chez Koltès, le lieu de toutes les contradictions" (Lazaidès, Jeu, 1999: 155).

(Le face à face verbal et la puissance ....) Dr. Alaaedin Baheidin Alaaedin 
Comme dans le cas du lieu, l'opposition dans le temps est concrétisée dans le passé représenté par de "là-bas" et le présent "ici", pour créer une liaison entre aujourd'hui et autrefois ou entre le passé et le présent:

"Monique- Autrefois il y avait des lampadaires, ici; c'était un quartier bourgeois, ordinaire, animé, je ne me souviens très bien. Il y avait des parcs avec des arbres; il y avait des voitures; il y avait des voitures et des commerces... Mais aujourd'hui, Seigneur! N'importe quel individu, le plus innocent, qui se perdrait là même en plein jour pourrait se faire massacrer en plein soleil..." (Koltès, Quai ouest, 1985: 13).

La contradiction qui se trouve entre le passé et le présent touche presque tous les personnages dans la pièce sauf Abad et Fak. Ils se trouvent au cœur du drame et continuent leur chemin. Koch et Monique viennent du monde de l'échange commercial, c'est-à-dire, le monde financier. Mais, à ce moment, ils doivent affronter le monde de la misère.

Donc, nous apercevons que la contradiction relevant des: "figures de style par rapprochement" (Fantanier, 1977: 377) attire l'attention par une opposition et a pour rôle: "d'exprimer des réalités complexe, ambiguës ou apparemment contradictoires." 
(Pougeoise, 2006: 176). Donc, nous pouvons dire, que l'opposition est comme: 1'"auxiliaire de l'ironie" (Morier, 1981: 830).

\section{$\underline{\text { V- Echange des paroles }}$}

\section{1) Echange des paroles creuses:}

En étudiant la puissance persuasive des paroles, nous devons commencer par l'interprétation de l'échange des paroles entre les interlocuteurs qui permet de comprendre non seulement le dialogue, mais aussi le silence qui se trouve dans la pièce, comme le silence d'Abad.

Nous remarquons la présence de la négation dans les paroles échangées des personnages, ce que nous pouvons considérer comme une forme textuelle. Cette négation est un élément essentiel de l'énonciation dans "Quai ouest".

La plupart des événements dans "Quai ouest" se trouve dans un conflit ou un duel. En effet, c'est un commerce, dans lequel, les personnages doivent faire une action d'échange, où ils échangent des objets. Tout le monde peut échanger n'importe quoi dans le hangar. A la suite de ces échanges, on peut arriver à l'échange des désirs de chaque personnage. Donc, c'est une chaîne de négociations qui: "circulent de main en main des objets qui se révèlent inutiles... mais porteur d'un échange symbolique, où s'exprime le désir de chacun." (Salino, 2009: 224). 
Dans "Quai ouest", il est à remarquer que ces échanges se passent à travers la confrontation, l'opposition, la dérision et le refus et l'insolence. Mais selon Herbert Paul Grice, qui croit que les personnages ou les interlocuteurs doivent essayer à arriver à un échange rationnel de paroles qui aboutit à la fin à faciliter la compréhension des énoncés, en disant que:

"La catégorie de quantité concerne la quantité d'information qui doit être fournie, et on peut y rattacher les règles suivantes:

1- Que votre contribution contienne autant d'information qu'il est requis.

2- Que votre contribution ne contienne pas plus d'information qu'il n'est requis." (Grice, 1979: 61).

Selon la parole de Grice, chaque personnage doit offrir l'information nécessaire, car l'information offerte n'est pas suffisante, cela aboutit à approfondir les malentendus entre les interlocuteurs. La parole de Grice qui concerne la fidélité du locuteur doit être sincère. Donc, le respect de ces bases aboutit à une compréhension mutuelle des énoncés lors de la conversation.

La pièce "Quai ouest" est caractérisée par un groupe de questions brèves posées à Koch. Ces questions sont destinées également aux spectateurs. Ceux-ci connaissent bien que ces questions n'auront pas de réponses, en même temps, ils s'imaginent être les récepteurs directs de ces questions qui ont 
besoin d'un interlocuteur, c'est pourquoi, c'est une pièce qui n'est qu'un: "désert d'une communication avortée avec autrui." (Barault, 1997: 104). Monique qui prétend s'adresser à Koch, essaie d'attirer l'attention du spectateur, dans ce cas, le spectateur et Monique sont réunis et l'espace tragique sans issue structure la pièce et son thème essentiel.

La pièce n'offre pas de renseignements sur l'action dramatique. Le début de la pièce pousse le spectateur à partager avec Monique ses sentiments, elle est séquestrée dans une obscurité profonde, elle ne voit rien et elle n'arrive pas à sortir de cette obscurité.

Il est à remarquer que cette obscurité qui enferme Monique est comme celle qui domine et couvre la salle de théâtre où se trouvent les spectateurs. Ceux-ci sont invités à partager la sensation de réalité malgré la distance qui sépare la scène de la salle de théâtre, mais qui diminue progressivement grâce au monologue de Monique.

D'autre part, c'est le monologue de Monique qui montre que nous sommes au "Quai ouest", c'est un espace de théâtre à l'instar de la salle de théâtre. A travers la description de cet espace au début de la pièce, nous pouvons comprendre les événements qui se succèdent en menant le spectateur dans une obscurité complète où se déroulent les événements dramatiques de la pièce.

Donc, nous pouvons remarquer que le titre de la pièce joue un rôle très important dans le déroulement dramatique. Ce lieu 
horrible est bien fermé que tous les personnages veulent quitter, mais personne n'arrive jamais, car l'issue unique, c'est la mort.

Dans "Quai ouest", nous ne pouvons pas négliger le monologue romanesque. Nous avons plusieurs monologues dans la pièce: le monologue de Fak, celui de Rodolfe et celui d'Abad. André Maïsette a expliqué la signification du monologue romanesque en disant qu'il est: "sans doute le plus original, le plus mystérieux aussi, pas le moins textuellement spectaculaire." (Maïsette, 2013: 184).

Nous remarquons que dans les monologues de Koltès que le discours des personnages n'est pas créé pour lui seul. Ce discours est destiné à un autre, soit absent, soit muet comme dans le cas d'Abad. A l'inverse du monologue conversationnel, nous trouvons dans les monologues koltésiens, un énonciataire qui est voulu et cherché par l'énonciateur; c'est-à-dire, un interlocuteur qui cherche un lien avec l'autre, à qui la parole s'est adressée.

Donc, la définition traditionnelle du monologue ne s'accorde jamais avec celui de Koltès, celui-ci a utilisé le monologue qui se caractérise par: "l'absence d'échange, qui permet de faire connaître les pensées d'un personnage... et qui est destiné au spectateur pour lui permettre de suivre l'évolution intérieure du personnage." (Vigeant, 1989: 155).

Dans la pièce, Charles prononce sa parole pour exprimer ses opinions dans un monologue, ainsi qu'Abad qui énonce ses paroles aux oreilles de Charles. Nous constatons qu'il y a un lien 
solide entre Abad et Charles et cela se manifeste dans la confiance qu'accorde Abad à Charles. Abad apparaît sur la scène sans aucune indication scénique qui précise sa présence. Mais, le spectateur voit Abad sur le plateau, tandis que le lecteur ne peut ressentir sa présence qu'après cette indication scénique: "Charles se dirige vers Abad" (Koltès, Quai ouest, 1985: 21). Le monologue d'Abad paraît ambigu pour les lecteurs:

"Qui est-tu? Celui qui a vu le diable, qui est-tu? J'essaie de le dire: je rentrais une nuit par le grand jardin avec le sac d'école sur le dos, je vis un homme sous le réverbère le dos tourné... puis il m'arracha mon nom et le jeta dans l'eau de la rivière avec les ordures... mais plus je le dis plus je le cache, c'est pourquoi je n'essaierai plus, ne me demandes plus que je suis. Dit Abad" (Koltès, Quai ouest, 1985: 19-20).

\section{2) Echange des paroles faibles:}

Les personnages dans "Quai ouest" n'arrivent pas à faire un véritable dialogue avec l'autre. Le dialogue pour les personnages n'est qu'un moyen pour la négation essentielle de leur vie. La communication entre les membres de la famille de Charles n'est pas correcte. Charles et sa sœur se querellent continuellement, 
parce que Charles veut convaincre sa soeur de rester chez elle avec leurs parents, et en même temps, elle l'implore de ne pas partir en utilisant tous les moyens possibles pour l'empêcher.

Quant à Cécile, la mère qui dit des mots sévères en rencontrant son mari Rodolfe et son fils Charles, ils échangent tous tout le temps des insultes et des menaces. Nous remarquons que leurs paroles se choquent et se coupent, sauf dans quelques scènes monologiques adressées à Abad qui ne fait qu'écouter les personnages, sans leur répondre.

Cet échange verbal négatif montre l'absence de la coopération dialoguée entre les interlocuteurs, cela se manifeste dans le dialogue entre Koch et Charles au cours de la première rencontre:

"La jetée. Au-dessus du fleuve flotte une

légère lumière blanche.

Entre Charles.

Sirène d'un bateau, au loin, étouffée.

Entre Koch, Envol d'oiseaux.

Koch (bas).- J'ai peur.

Charles (bas).- Pourquoi?

Koch- J'ai peur. Je ne sais pas pourquoi.

Charles- Tu as ton arme?

Koch- Une arme? Non. Pourquoi?..."

(Koltès, Quai ouest, 1985: 20). 
Nous remarquons dans ce dialogue l'abondance des questions courtes. "Pourquoi?", dans la première question qu'on utilise toujours pour connaître la cause et la réponse montre l'ignorance de Charles sur la raison de la peur de Koch. Tandis que les questions brèves "Une arme?", "Non. Pourquoi?", montrent que Koch ignore totalement le but de ces questions posées par Charles.

Ces échanges verbaux entre les deux interlocuteurs démontrent que ceux-ci appartiennent à des milieux sociaux opposés, ils n'arrivent jamais à se comprendre. Nous découvrons l'abîme entre les deux interlocuteurs qui a causé ce dialogue décousu. Le dialogue suivant montre cette rupture entre les deux personnages:

"Koch-Prenez la voiture.

Charles- Tu ne m'as pas donné d'argent.

Koch- Je vous ai donné mes cartes de crédit.

Charles- Pas d'argent. Koch- Mais c'est

l'argent, ça; je ne connais pas d'autre forme d'argent... Les billets et les pièces, c'est l'argent du pauvre, de l'argent du sauvage. Mes cartes de crédit sont de l'argent." (Koltès, Quai ouest, 1985: 23-24). 
Cette discontinuation dans la conversation montre l'ignorance de Charles de l'utilisation des cartes de crédit, ce qui montre la divergence en ce qui concerne le système économique. Cette divergence entre le deux interlocuteurs crée une communication décousue, ainsi qu'une rupture entre eux.

\section{3) Disputes stériles:}

Dans "Quai ouest", le couple Koch et Monique se disputent dès le début de la pièce jusqu'à la fin, ils se disputent verbalement:

"Monique- Dites-moi donc dans quel trou vous préférez qu'on tombe. Entre Koch.

Koch- Je sais-moi, très exactement où je suis.

Monique-Très exactement, tiens donc, vous êtes fort, très exactement, bravo. Débrouillez-vous tout seul puisque vous savez tout très exactement...

Koch- Ne risquez rien du tout, Monique, rentrez.

Monique- Rentrez? Comment voulez-vous que je rentre? J'ai les clés de la voiture..." (Koltès, Quai ouest, 1985: 12). 
En répondant aux questions désespérées de Monique, Koch donne une réponse impersonnelle "Je sais, moi, très exactement où je suis", ce qui pousse Monique de se fâcher et s'obstiner à remettre en cause ses mots vides de sens. Les réponses de Koch ne donnent aucune satisfaction à Monique qui accuse Koch de mentir et de ne pas dire la vérité, en lui adressant des reproches courtes comme "Rentrez?", "vos moyens?". Ces reproches interrogatifs sans réponses visent à montrer le manque de fidélité dans les répliques de Koch. Cette absence de fidélité envers Monique est concrétisée dans le désir de Koch d'éviter toute communication avec elle, car Koch était occupé par la question de son suicide. Ce manque de communication entre les deux interlocuteurs n'est qu'un des traits essentiels des dialogues dans "Quai ouest".

Koch doit affronter cette obscurité qui l'entoure, cette obscurité est pleine de gens qui cherchent à le maintenir vivant pour profiter de lui. Koch paraît troublant pour ces gens qui l'entourent. Sa présence entraîne plusieurs événements qui immergent le monde du quai dans une obscurité profonde:

"L'arrivée de Koch dans l'univers de Quai ouest est sans doute l'événement qui ébranle l'équilibre entre Abad et Charles. En cherchant la mort (le non-être) parmi les ombres de Quai ouest, Koch provoque une crise." (Hufmann, 1999: 79). 
Nous pouvons dire que dans "Quai ouest", Koch cherche: "cette passivité, un type de repos du monde." (De Oliveira, 2015: 292), Koch a voulu: "faire face à l'exposition publique de son échec... Choisir la mort dans un coin abandonné, oublié, c'est une façon de fuir cette exposition." (De Oliveira, 2015: 292), car pour Koch, la mort est un véritable état de passivité et d'inconscience; il a cherché tellement ce moment de repos et de paix.

Un autre couple dans "Quai ouest" représenté par Fac et Claire, ce couple concrétise la plaisanterie et l'humour dans la pièce. C'est un couple comique, leurs dialogues sont marqués par le détournement, ils ont leur langage, et usent des moyens persuasifs. Nous remarquons également que les paroles échangées entre Fak et Claire se font dans l'absence totale du respect des règles de coopération conversationnelle, cela se manifeste clairement dans le dialogue suivant:

"Fak- Tu es venu jusqu'ici, maintenant passe là-dedans.

Claire-Il fait bien trop noir là-dedans pour que je passe.

Fak- Il ne fait pas plus noir là-dedans qu'ici.

Claire- Eh bien justement, ici, il fait complètement noir. 
Fak-Il ne fait pas complètement noir ici puisque je te vois.

Claire-Et moi je ne te vois pas, pour moi il fait complètement noir donc." (Koltès, Quai ouest, 1985: 25).

Dans cet extrait ci-dessus, nous remarquons que malgré que ce couple se rencontre pour la première fois, ils s'opposent sans cesse. Dans ce dialogue, chaque personnage est convaincu tout à fait que son locuteur n'est pas coopératif dans la communication. D'ici, ce refus répété de la part de Claire "Il fait complètement noir", fait croire qu'elle a deviné l'intention de Fak. Devant ce refus obstiné de Claire, Fac déclare que l'obscurité n'est pas totale là-dedans, ce qui montre l'opposition complète de Claire.

Donc, la question de dispute entre les deux interlocuteurs est restreinte concernant la problématique de l'obscurité qui parait clairement dans leur conversation.

\section{4) La persuasion manipulée de Fak:}

Dans le dialogue entre Fak et Claire, celle-ci a montré une grande résistance face à la puissance persuasive de Fak qui est obligé de changer sa stratégie pour convaincre Claire, il commence par faire un commerce d'objets. 
Le commerce commence au moment où la persuasion verbale échoue. Mais, de nouveau, cette négociation verbale se heurte au refus permanent de Claire. Fak déploie ses efforts en utilisant une stratégie de manipulation afin que Claire se rallie à sa parole:

"Fak- Je le donne si tu passes avec moi làdedans.

Claire-(retirant sa main)-Alors non, je ne prends pas. Quand on donne quelque chose, on le donne et c'est tout, on ne demande pas autre chose, tiens.

Fak- Je ne demande rien, justement.

Claire-Comment ça, tu ne demandes rien?

C'est trop fort." (Koltès, Quai ouest, 1985: 29).

Les procédés de manipulation et adoptés par Fak consistent essentiellement sur la négation en essayant de réfuter la parole de Claire. L'opposition entre les deux interlocuteurs provient de l'ambiguïté langagière de "quelque chose" qui représente un: "paralogisme relevant d'un jeu d'ambiguïté portée par la construction syntaxique." (Hage, 2001: 86). Malgré les efforts déployés par Fak pour persuader Claire, celle-ci ne paraît pas se résigner à cette tentative de persuasion en disant: "mon frère me tabassera" (Koltès, Quai ouest, 1985: 29). 
Dans la confrontation verbale entre Fak et Claire, Fak apparaît comme un sophiste, sa parole avec Claire est chargée par des argumentations et de la négation comme s'il participait à un duel verbal, cela se manifeste clairement dans l'extrait suivant:

"Fak- Moi aussi, tu peux voir, je suis complètement propre toujours et n'importe où.

Claire: Comment ça, que tu serais propre? Cela, je ne le goberai pas. Tu viens de dire toi-même que tu ne te débarbouilles jamais, ce n'est pas moi qui l'ai dit, tiens.

Fak- Justement, ceux qui ne sont jamais débarbouillés depuis qu'ils sont tout petits ils sont toujours propres, parce que la crasse se désintéresse d'eux et leur glisse dessus." (Koltès, Quai ouest, 1985: 56).

Dans le dialogue ci-dessus, Claire se transforme en une femme offensive en adressant des reproches à Fak et commence à l'accuser. Face à cette accusation de la part de Claire, Fak se défend comme un sophiste en réfutant la parole de Claire et crée en même temps l'effet comique. Michel Dufour explique la définition du sophisme en disant:

"Le prestigieux grammairien Dumarsais définit les sophismes comme des 
raisonnements éblouissants dont on sent

bien la fausseté." (Dufour, 2012: 305).

Cet effet comique provenant des preuves vaines ou des paroles sophistes de la part de Fak aide à créer un équilibre entre le tragique et le comique dans la pièce, c'est un élément nécessaire dans l'écriture dramatique de Koltès. Fak est le personnage qui prend en charge le rôle du joueur comique qui offre des idées sans principes.

Donc, nous pouvons considérer la négation chez Fak comme un élément essentiel de communication avec Claire. Cette négation comporte le refus des présupposés conversationnels et les preuves vaines qui forment l'énonciation. Ses paroles négatives ne sont qu'une forme du refus de l'autre et cela s'accorde avec le caractère conflictuel de Fak.

Quant à Claire, celle-ci essaie de réfuter tout essai de Fak, mais elle se heurte toujours à son opposition:

"Claire: En plus, je sais précisément pourquoi tu veux que je passe là-dedans; et pour cela, je ne veux pas de cela, car je sais très précisément de quoi il s'agit.

Fak-Si tu es encore petite, tu ne peux savoir très précisément pourquoi je veux qu'on passe tous les deux là-dedans, et si tu savais précisément pourquoi on y passerait, alors, tu n'es pas si petite que ça, ne fais pas tant 
d'histoires, passe et voilà tout." (Koltès, Quai ouest, 1985: 26).

Généralement, le dernier dialogue est dominé par Fak, parce qu'il a la puissance de déformer les preuves de Claire, en posant des questions qui attaquent les côtés faibles dans ses paroles. Face à cette argumentation vaine de la part de Fak, Claire se trouve la victime de la manipulation de Fak. Elle révèle son impuissance oratoire en face de Fak et cela aboutit à sa complète défaite dans la confrontation verbale avec lui.

D'autre part, Claire essaie de montrer un peu de résistance face à Fak, mais celui-ci utilise tous les moyens possibles d'argumentation qu'il possède pour convaincre Claire:

"Fak-Mais, s'il te plaît, tu pourrais savoir comment c'est bien ou pas bien puisque tu n'as jamais essayé cette chose-là avec personne? Et que, si tu avais essayé et que tu dirais: ce n'est pas bien du tout, je dirais alors: tans pis, on ne passe pas..." (Koltès, Quai ouest, 1985: 27).

En bref, dans le discours des deux interlocuteurs, Fak essaie de montrer la contradiction chez Claire, entre sa minorité et l'âge légale. Sur cette contradiction est basée la puissance persuasive de Fak. Chaïm Perelman explique cette contradiction qui: "consiste en deux assertions entre lesquelles il faut choisir, à

(Le face à face verbal et la puissance ...) Dr. Alaaedin Baheidin Alaaedin 
moins de renoncer à l'une et l'autre." (Perelman et Olbrechts Tyteca, 2008: 263).

\section{Abad: symbole de refus du dialogue}

Dès le début de la pièce, le personnage d'Abad se trouve au cœur du drame. Il se trouve au début et au dénouement, et peutêtre avant le début de la pièce, c'est-à-dire avant le lever du rideau, Koltès affirme qu' "Abad n'est pas un personnage en négatif au milieu de la pièce; c'est la pièce qui est le négatif d'Abad." (Koltès, in Quai ouest, 1985: 168). Abad est un personnage à la peau noire, Koltès l'a utilisé pour symboliser la politique raciale, Abad avec sa couleur représente un signe de son être, c'est pourquoi Koltès: "en introduisant Abad dans le hangar, Charles invite l'ombre à s'établir dans l'univers de Quai ouest." (Hufmann, 1999: 74).

Donc, nous pouvons considérer que la présence de ce personnage dans la pièce est un symbole du malheur, c'est: "une figure typiquement koltésienne" (Benhamou, 2014: 24). Dans "Quai ouest", tous les personnages connaissent qu'Abad n'est ni muet ni sourd. Nous remarquons qu'Abad parle à l'oreille de Charles et celui-ci rapporte ses paroles aux autres. Ce personnage ambigu n'existe sur scène que par des gestes et des actions qui causent la mort de Koch et Charles. Abad reste silencieux jusqu'à la fin de la pièce. Donc, nous pouvons dire que le silence d'Abad représente la volonté de refuser le dialogue Jean-Pierre Sarrazac voit qu'on doit: "enregistrer le silence qui monte des corps; puis il 
lui faut transpercer ce silence afin de pouvoir le transcrire, le transposer en lui conférant sa plus haute expression théâtrale... le vrai silence est bruant et possède plus d'un trop-plein que d'une absence de mots." (Hotte, 1988: 110).

Avec ce mutisme, Abad devient un caractère énigmatique. Il devient un personnage dangereux et ambigu: "Abad, par son mutisme, en vient à façonner la parole de ses interlocuteurs qui, loin de se confier dans l'espace intime d'un soliloque, se livrent, sans prudence, aux révélations souvent agressives de leurs impulsions secrètes." (Poujardieu,2003: 56).

La présence d'Abad est partout sur la scène. Comme le hangar qui inclut les personnages, Abad les guette dans le hangar et les pousse vers une fin tragique. Selon Anne Ubersfield, le mutisme d'Abad représente: "une forme d'angoisse dont la présence muette et finalement meurtrière d'Abad, dans Quai ouest, est la brutale métaphore... que l'Autre menace de mort et de vie." (Ubersfield, 1999: 143).

Quant au nom d'Abad, nous remarquons qu'il n'a pas de sens, les spectateurs n'entendent pas son nom. Nous remarquons que ce nom ne se trouve que dans les indications scéniques. Dans la pièce, on l'appelle en utilisant: "négro", "mon vieux", "vous", "tu". Il est à remarquer que le nom d'Abad n'est utilisé que pour 
les lecteurs, c'est un personnage ambigu qui: "échappe à la fixation qui lui imposerait l'expression orale ou toute caractérisation langagière." (Meuré, 2007: 218-219).

Ainsi, Abad était tout loin de toute question langagière. Le silence d'Abad est à l'instar du hangar déserté qui domine et précise la destinée des personnages sans paroles comme Abad. En ce cas, Abad ressemble au hangar qui conduit les personnages vers une fin catastrophique:

"... il est aussi le récepteur de toutes leurs

demandes et le facilitateur de la réflexion des autres simplement en écoutant leurs monologues." (Guenther, 2010: 6).

Dans "Quai ouest", les personnages dialoguent, ils parlent et Abad est celui qui écoute, Anne Ubersfield voit que: "c'est à ce personnage (Abad) que sont adressés les quasi-monologues. Il est l'Autre par essence, le récepteur de la demande; il est le grand Ecouteur." (Ubersfield, 1999: 158).

Dans la pièce, Abad est comme un destinataire, reçoit non seulement les insultes des personnages, mais aussi leurs demandes urgentes car: "parler, c'est anticiper le calcul interprétatif de l'interlocuteur." (Kerbrat-Orecchioni, 1990: 25).

Donc, Abad est un récepteur des paroles des autres, il accepte toutes les paroles et ne refuse jamais tout ce qu'ils disent. Il accueille les paroles, ainsi que les secrets des autres personnages. 


\section{Conclusion}

Ce qui caractérise le théâtre de Marie-Berbard Koltès, c'est la parole désespérée. Le théâtre de Koltès n'est qu'une tragédie langagière où la parole n'est pas un élément supplémentaire de l'action, mais inversement, elle représente l'espace de cette action et l'arme destructive des personnages dans leurs confrontations. Nous remarquons dans la pièce "Quai ouest" que tous les personnages possèdent cette arme et construisent leurs propres paroles en utilisant des procédés linguistiques qui s'accordent avec les circonstances au cours d'une analyse rhétorique persuasive qui révèle la relation avec l'Autre et avec le monde entier.

Notre étude a commencé par une supposition que le théâtre koltésien repose essentiellement sur l'affrontement des personnages et que tous les discours langagiers écrits ou oraux sont considérés comme discours persuasifs, car ils visent à influencer le destinataire. Toutes les paroles visent à agir sur l'autre et à changer sa façon de penser. Chaque parole force l'autre à croire, à faire ou même à changer son système de réfléchir.

Il est à remarquer que Koltès a créé une relation solide entre la salle et la scène en créant une communication scénique où le spectateur devient l'allocutaire à côté de l'allocutaire principal qui se trouve sur la scène pour diminuer la distance entre la salle et la scène et rapprocher les spectateurs de la scène pour faciliter la question de la persuasion à travers ce contact entre eux. 
C'est pourquoi, le thème de l'échange verbal est primordial dans le théâtre de Koltès. Dans son écriture dramaturgique, tout devient échangeable en utilisant la parole comme un moyen de négociation.

Dans la pièce "Quai ouest", l'échange verbal a été représenté de façon très claire concernant les objets matériels, ainsi que le déroulement des négociations entre les interlocuteurs.

Malgré ces négociations matérielles permanentes, les personnages n'ont pas réussi à arriver à leur but. Cette insatisfaction approfondit chez eux ces échanges verbaux qui les mènent à la violence physique à la fin de la pièce.

Donc, le thème de l'échange se lie étroitement à celui de la violence. L'échange conversationnel structure le nœud de la pièce, cela montre qu'il y a un lien fort entre les actions dramatiques et les paroles des personnages dans la pièce.

En commençant leurs négociations, les personnages dans "Quai ouest" ont utilisé tous les procédés techniques et persuasifs, parmi lesquels; les arguments vains et les menaces. Mais tous les efforts restent sans écho, car ils désobéissent aux règles concernant la communication.

L'arrêt et l'échec de l'échange verbal sont dus à la désobéissance aux règles de communication, mais ils révèlent les désirs des personnages. Ces désirs cachés que les interlocuteurs expriment par des paroles vives comme celles de Rodolfe et 
Cécile ou par les trois monologues d'Abad qui reste muet dans la pièce.

Etant observateur et écouteur de toutes les paroles des personnages, Abad reste le seul personnage qui se trouve loin de ces négociations, car il n'a pas besoin de l'autre. Ce personnage ambigu ressemble beaucoup au hangar déserté qui représente l'espace de la confrontation entre deux classes sociales représentant deux groupes tout à fait opposés. Abad comme le hangar pousse les personnages vers une fin tragique et inéluctable, sans dire un seul mot.

Finalement, l'abîme profond entre les deux groupes opposés et leurs confrontations verbales sont représentées à travers des procédés rhétoriques pour mettre en relief le monde capitaliste où dominent les contrastes. 


\section{Bibliographie}

\section{I- Corpus}

1- KOLTES Marie-Bernard, "Quai ouest", Paris, Les Editions de Minuit, 1985.

\section{II- Ouvrages consacré à Koltès et son ouvre}

1- AUGE Mark, Non-lieux, Introduction à une anthropologie de la surmodernité, Paris, Edition du Seuil, 1992.

2- BENHAMOU Anne-Françoise, Koltès dramaturge, Besançon, Les Solitaires intempestifs, coll. "Du désavantage du vent", 2014. 3- BERNARD Florence, Koltès, une poétique des contraires, Paris, Editions Champion, 2010.

4- BRUSCHI Flippo, Le style monologique dans l'Héritage, Quai ouest et Le Retour au désert, in André Petitjean, "Bernard-Marie Koltès. Les registres d'un style", Editions universitaires de Dijon, 2014.

5- DUFOUR Michel, Argumenter. Cours de logique informelle, Paris, Armand Colin, 2012.

6- FONTANIER Pierre, Les Figures du discours, Paris, Flammarion, 1977.

7- HAGE Samar, Bernard-Marie Koltès. L'esthétique d'une argumentation dysfonctionnelle, Paris, L'Harmattan, 2011.

8- HIRSCHMULLER Sarah, Le jeu du désir, in Christophe Bident, "Voix de Koltès", Carnets Séguier, 2004.

9- KERBERT-ORECCHIONI Caherine, Les Interactions verbales, Paris, Armand Colin, 1990. 
10- KOLTES Bernard-Marie, Une part de ma vie: entretien 1983-1989, Paris, Les Editions de Minuit, 1999.

11 , Pour mettre en scène Quai ouest, in "Quai ouest", Paris, Les Editions de Minuit, 1985.

12- MAÏSETTI Artaud, Koltès, écrire. La trace, la mort, la survie: Quai ouest (le texte), in Matthieu Méval (dir.), 'La Littérature théâtrale, Entre le livre et la scène", Montpellier, Editions l'Entretemps, 2013.

13- MEURE Christph, La didascalie- écran: Bernard-Marie $\underline{\text { Koltès }}$, in Florence Fix et Frédérique Toudoire-Surlapierre, "La Didascalie dans le théâtre du XXe siècle. Regarder l'impossible", Editions universitaires de Dijon, 2007.

14- MORIER Henri, Dictionnaire de poétique et de rhétorique, Paris, PUF, 1981.

15- PERELMAN Chaïm et OLBRECHTS Tyteca, Traité de l'argumentation: la nouvelle rhétorique, Edition de l'Université de Bruxelles, 2008.

16- POUGEOISE Michel, Dictionnaire de poétique, Paris, Edition Belin, 2006.

17- ROBRIEUX Jean-Jacques, Eléments de rhétorique et d'argumentation, Malakoff, Dunod, 1993.

18- RYNGAERT Jean-Pierre et SERMON Julie, Koltès et ses personnages ou: de que l'auteur est-il le contemporain? In André Petitjran (dir.), "Bernard-Marie Koltès: Textes et contextes", Université Paul Verlaine- Metz, 2011. 
19- SALINO Brigitte, Bernard-Marie Koltès, Paris, Editions Stock, 2009.

20- SARRAZAC Jean-Pierre, Jeux de rêves et autres détours, Circé, 2004.

21- SEBASTIEN Marie-Paul, Bernard-Marie Koltès et l'espace théâtral, Paris, L'Harmattan, 1999.

22- UBERSFIELD Anne, Les termes clés de l'analyse du théâtre, Paris, Seuil, 1996.

23Bernard-Marie Koltès, Arles, Actes Sud, coll. "Apprendre", 1999.

24- VIGEANT Louise, La lecture du spectacle théâtral, Laval: Mondia Editeurs, 1989.

\section{III- Articles de revues}

1- BRAULT Pascal-Anne, "Bernard-Marie Koltès: théâtre et vérité", in Romance Notes, vol. 38, No. 1, University of North Carolinat at Chapel Hill for its Department of Romance studies, 1997.

2- CHEREAU Patrice, "Koltès, combat avec la scène", in Théâtre d'aujourd'hui, No. 5, 1996.

3- DE OLIVEIRA Uendel, "Le suicide dans la dramaturgie de Bernard-Marie Koltès: Une étude des personnages", in Passage de Paris, No. 10, 2015, pp. 285-300.

4- GRICE Herbert Paul, "Logique et conversation", traduit par Frédéric Berthet et Michel Bozon, in Communication, No. 30, 1979.

(Le face à face verbal et la puissance ...) Dr. Alaaedin Baheidin Alaaedin 
5- GUENTHER Melissa, "L'étranger dans le théâtre de BernardMarie Koltès: Quai ouest et La Nuit juste avant les forêts", in Interfrancophonies, No. 3, 2010, pp. 1-14.

6- GUIBERT Hervé, "Comment porter sa condamnation", entretien avec Bernard-Marie Koltès, in Le Monde, 17 février 1983.

7- HOTTE Véronique, "Des histoires de vie et de mort", entretien avec Koltès, in Théâtre/Public, No. 84, novembre-décembre 1988. 8- HUFMANN shawn, "La texture lumineuse de Quai ouest de Bernard-Marie Koltès: le cas de l'ombre", in L'Annuaire théâtral, No. 26, 1999, pp. 69-83.

9- MEREUZE Didier, "Entretien avec Patrice Chéreau", paru sous le titre de "Il faut apprendre à cheminer avec l'auteur", in Alternatives théâtrales, juin 1990, No. 35-36.

10- POUJARDIEU François, "La figure du noir dans la dramaturgie de Bernard-Marie Koltès", in Théâtre/Public, No. 168, mai-juin 2003.

11- PURKHARDT Brigitte, "Bernard-Marie Koltès et la face cachée du désespoir", in Jeu, No. 87, 1998, pp. 71-98.

12- VIGEANT Louise, "Bernard-Marie Koltès: Les contours infranchissables de la solitude", in Jeu, 1990, No. 57, pp. 35-40. 


\section{ملخص البحث}

" المواجهة الثفهية والقدرة الإقناعية في مسرحية الرصيف الغربي للكاتب برنار

$$
\text { ماري كولتيس" }
$$

ولد برنار ماري كولتيس في عام 91 19 في مدينة ميتز وتوفي في عام 919 امبرض الايدز عن عمر اءـ عام. لقد قام بكتابة ست مسرحيات هامـة وكانت حياته الادبيـة التي استمرت مـا يقارب العشر سنوات كافية أن يشغل اسمه العالم قاطبة ولا سيما في وسط عـالم المسرح. ويمثل مسرحه تجربـه او محاولة مستمرة من اجل خلق نوع مـن

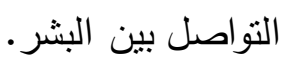

فدراستتا ترتكز على الحوارات المليئة بالمواجهات الثفهية واللغويـة في ضوء البلاغـة الإقناعية. وسنقوم كذللك بمعالجة موضدوع الصمت لنخصية عبـاد والذي لم يكن في الواقع أصما ولا أخرسا ولكن لايتقوه مطلقا على خشبة المسرح ولكن من المدهش في تلك المسرحية أن معظم الاشخاص لا يتكلمون مـع عباد، لأنـه يقر كلامهم ولا يرفضـه ابدا. فهذا الخرس له دور رمزي في المسرحبة والذي سيكون هو الموضوع الرئيسي في 